\author{
Maestría en Economía \\ Facultad de Ciencias Económicas \\ Universidad Nacional de La Plata
}

TESIS DE MAESTRIA

\begin{abstract}
ALUMNO
Diego Margot

TITULO

Rendimientos de la Educación en la Argentina: Un Analisis de Cohortes

DIRECTOR

Walter Sosa Escudero

FECHA DE DEFENSA

$3 / 30 / 2001$
\end{abstract}




\section{Rendimientos de la educación en Argentina: Un análisis de cohortes}

\section{Introducción}

A partir del trabajo de Gary Becker (1964) se ha desarrollado considerablemente una rama de la literatura que ve la decisión de educarse como un proyecto de inversión. El activo en el que se invierte es capital humano, y las herramientas analíticas utilizadas para estudiar este problema son similares a las utilizadas para el caso del capital físico. Esta literatura generó un cambió en el foco de atención de numerosos problemas de economía laboral: la variable empírica de interés dejó de ser el salario actual, observado en un momento del tiempo, y pasó a ser el ingreso obtenido a lo largo de toda la vida laboral. La teoría del capital humano se focaliza en el ingreso permanente antes que en el ingreso corriente (Rosen 1977).

Al igual que la decisión de invertir en capital físico, la decisión de invertir en capital humano posee costos y beneficios. El criterio básico para evaluar la decisión de ambos tipos de inversión es el mismo: si los beneficios del proyecto superan a los costos, conviene llevar a cabo la inversión. La variable más usada para evaluar la inversión en capital humano ha sido la tasa interna de retorno. Esto se debe a que es fácil de calcular, ya que por su naturaleza el capital humano no puede ser observado en mercados de trabajo libres, en donde no hay un precio que refleje el capital humano que posee un individuo (si puede existir este precio en mercados con esclavitud) y a que no hay una tasa de interés obvia para descontar a los flujos de este tipo de inversión, debido a que no se encuentran inversiones similares en cuanto a características del proyecto y riesgo del mismo.

Una persona que ha alcanzado un cierto nivel educativo tiene dos opciones: entrar al mercado laboral, y obtener una secuencia de ingresos $\left\{\mathrm{y}_{\mathrm{t}}\right\}$ a partir del momento $\mathrm{b}$, o continuar estudiando por e años y obtener una secuencia de ingresos $\left\{x_{t}\right\}$ a partir del momento $t+e$. Supongamos que $x_{t}>y_{t}$ para todo $t>t+e, y$ que $x_{t}<y_{t}$ para $t \leq t+e$, es decir, existe un costo que es necesario incurrir para obtener mayores ingresos en un futuro. De este modo el problema no tiene solución trivial. Como los costos y beneficios se distribuyen a lo largo del tiempo, para evaluar la conveniencia de llevar a cabo el proyecto hay que expresar los costos y beneficios del mismo en términos homogéneos. Si la tasa de interés es cero, simplemente se comparan la suma de los costos con la suma de los beneficios. Pero si la tasa de interés es positiva, los agentes prefieren un peso hoy a un peso mañana, y por lo tanto hay que descontar los flujos correspondientes a costos y beneficios. La tasa de interés que iguala a cero la sumatoria (descontada) de costos y beneficios es la tasa interna de retorno asociada al proyecto. Esta tasa interna de retorno, i, debe compararse con la tasa de interés de mercado para un proyecto con igual riesgo, $\mathrm{r}$. Si i > r, conviene realizar el proyecto.

Para estimar la tasa interna de retorno del proyecto de invertir en capital humano (educarse) hay que conocer los ingresos de un individuo con un cierto nivel de educación a lo largo de su vida, y compararlos con los ingresos de otro individuo de similares características, pero con un nivel de educación inferior. Así se pueden conocer los diferenciales de ingresos (el premio por una mayor educación), descontarlos y calcular el retorno del proyecto. 
Hay dos métodos para estimar este retorno. El primer método es el "estático". Este método utiliza datos de un solo momento del tiempo (es un cross-section) y para obtener el perfil de ingresos de una persona durante toda su vida, los infiere de los ingresos de otras personas con las mismas características. La inferencia es inevitable, ya que al ser un cross-section, se tienen datos de un solo momento de la vida de esta persona, y hay que completar el perfil de ingresos. Numerosos trabajos han realizado un estudio de los retornos a la educación empleando esta metodología: Lachler para México (1993), Psacharopoulos para Venezuela (1995), Hartoog et al. (1996) para Portugal, Maani para Nueva Zelandia (1997), Moock, Patrinos y Venkataraman para Vietnam (1998). Para Argentina, un trabajo de Carola Pessino (1995) analiza los retornos a la educación en el Gran Buenos Aires en 1986 y 1993.

El segundo método es el "dinámico". Este método utiliza series de tiempo, y de esta forma se puede obtener el perfil de ingresos de una persona a partir de los ingresos observados para la persona analizada en distintos momentos del tiempo. La principal ventaja de esta forma de calcular los retornos a la educación es que no es necesario inferir los ingresos, como en el método anterior. Como se explica en la sección 2, esto evita la introducción de varios supuestos, y hace que en este sentido la estimación por el método dinámico sea más general. Los trabajos que emplean la metodología dinámica son menos abundantes. Japelli (1999) la emplea en un estudio para Italia, Card y Lemieux (2000) usan este método para analizar si en EE.UU. la caída en la oferta laboral de jóvenes universitarios puede explicar el aumento en el retorno de estos trabajadores. Arias y Mac Mahon (1999) utilizan el método dinámico para estimar retornos a la educación en EE.UU. El principal objetivo de este trabajo es estimar los retornos dinámicos a la educación para Argentina.

El carácter del trabajo es esencialmente empírico. Se pretende, a partir de los datos provistos en las encuestas de hogares, hallar las tasas de retorno dinámicas para distintos niveles educativos, para la región del Gran Buenos Aires, y compararlas con las tasas de retorno estáticas. El período comprendido por este estudio es de veinticinco años, desde 1974 hasta 1999, que es el período más prolongado para el que se cuenta con información completa proveniente de encuestas de hogares.

En la sección 2 se presentan las distintas formas de estimar los retornos a la educación. En la sección 3 se describen las cuestiones empíricas: fuentes de información, el manejo de los datos y los problemas que surgieron para armar las variables empleadas en el análisis. En la sección 4 se muestra la evolución de los ingresos laborales de las personas ocupadas, se analiza en detalle la forma de calcular los retornos a la educación por el método de tasas de retorno dinámicas y se calculan los costos y beneficios de invertir en cada nivel educativo. Posteriormente se interpretan los resultados obtenidos y se realiza una comparación con los resultados encontrados a través de la metodología de Mincer (Mincer 1974). Para finalizar, en la sección 5 se presentan las conclusiones. 


\section{Retornos estáticos vs. Dinámicos}

\subsection{La decisión de educarse como proyecto de inversión}

La inversión en capital humano aumenta la productividad de la persona que realiza la inversión. Esta idea ha estado por años en la literatura (Marshall 1948) ${ }^{1}$. Ben Porath (1967) plantea el problema desde la perspectiva de una persona que busca maximizar su utilidad intertemporal y debe decidir cuánto tiempo dedicar a la inversión en capital humano. Adison y Siebert (1991) formalizan estas ideas y las aplican al mercado de trabajo. La teoría postula que las empresas que maximizan beneficios estarán dispuestas a pagar un mayor salario para contar con los servicios de este trabajador. Los beneficios, entonces, radican en los mayores salarios que se obtienen respecto a los que no realizaron inversiones en educación, cuando el trabajador ingresa al mercado laboral. Los costos están representados por los salarios no percibidos durante el período en el que se realiza la inversión, y por costos directos asociados a la inversión: gastos en materiales necesarios y matrículas, en el caso de que la educación no sea gratuita. Los agentes van a invertir hasta que el costo marginal de la inversión se iguale a los beneficios marginales. El costo de invertir un año adicional en educación es el salario anual, más los costos directos de la educación. El beneficio del año adicional de educación está formado por la suma de los diferenciales de salario anuales para el resto de la vida laboral. Esta condición lleva a la siguiente igualdad (en donde se supone que los costos directos son nulos):

$\mathrm{W}_{0}=\sum \mathrm{j}\left(\mathrm{W}_{1}-\mathrm{W}_{0}\right)(1+\mathrm{r})^{-\mathrm{j}}$

en donde $\mathrm{W}_{0}$ representa el salario de un trabajador con (n-1) años de educación, $\mathrm{W}_{1}$ representa el salario de un trabajador con $\mathrm{n}$ años de educación y $\mathrm{r}$ es la tasa de interés relevante para el agente.

\section{Gráfico $\mathrm{N}^{\circ} 1$}

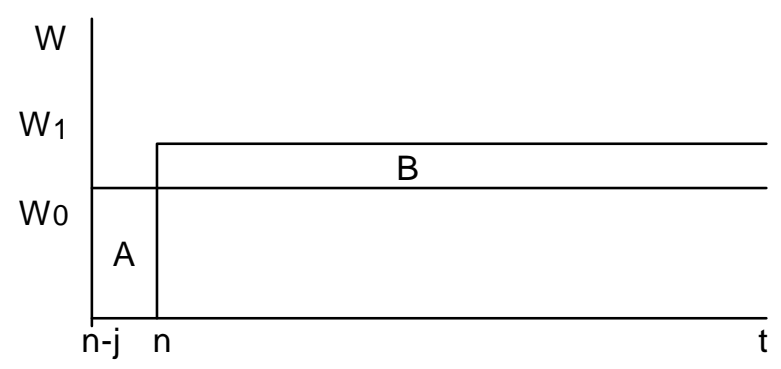

En el gráfico 1, los costos marginales están representados por el área $\mathrm{A}$, y los beneficios marginales por el área B. Se supone para mayor claridad que los salarios, para ambos niveles de educación, son constantes en el tiempo, pero los resultados no dependen de este supuesto. De acuerdo a la ecuación 1, las áreas A y B (descontada) deben ser iguales.

La ecuación 1 se puede computar si se cuenta con los perfiles de salarios para dos grupos de individuos con distinto nivel educativo, y una tasa de interés. De esta forma se puede calcular el valor presente neto de estudiar $\mathrm{j}$ años adicionales. Alternativamente, se puede computar la tasa interna de retorno (TIR), es decir, cual es el valor de $r$ que

${ }^{1}$ Citado en Rosen (1977) 
resuelve (1) y compararlo con la tasa de interés de mercado. El método de la TIR encuentra la tasa de interés que hace igual a cero el valor presente neto del flujo de fondos compuesto por los costos de educarse y los diferenciales de salarios que reciben los trabajadores más educados. Son dos métodos alternativos para evaluar la conveniencia (o no) de encarar el proyecto. La aplicación no es tan directa ya que ambos métodos poseen sus dificultades. Para calcular el valor presente neto hay que suponer una estructura temporal de tasas de interés, y hay que determinar qué tasa de interés es relevante para el agente que toma la decisión. El problema se suele simplificar postulando que la tasa es la misma para todos los individuos, pero esto no siempre es correcto. Como se verá más adelante este punto se relaciona con la igualdad de oportunidades. Cuando se calcula la tasa interna de retorno, se resuelve una ecuación de grado mayor a uno y por lo tanto, puede tener más de una solución para r. Por otra parte, bajo ciertas estructuras de los diferenciales de salarios ${ }^{2}$ puede ocurrir que los métodos de VPN y TIR den respuestas distintas ante la opción de invertir j años adicionales en educación.

Debido a que ningún método presenta ventajas claras, en este trabajo se utilizará el criterio de la tasa interna de retorno, porque es el criterio usado con mayor frecuencia en la literatura y es útil a los fines de comparación de los resultados.

\subsection{Dos criterios para evaluar la inversión en educación: estático y dinámico}

Los trabajos empíricos sobre el tema tuvieron un gran desarrollo a partir del estudio de Jacob Mincer (1974). Mincer estimó los retornos a la educación por el método estático. El enfoque de Mincer consiste en estimar a través de una regresión por el método de mínimos cuadrados ordinarios la siguiente ecuación:

$\ln y_{i t}=a+b e e d u c_{i}+c \exp _{i t}+u_{i t}$

En donde ln $y_{i t}$ es el logaritmo natural de los ingresos del individuo $i$ en el año t, educ ${ }_{i}$ es una variable que refleja el nivel educativo del individuo $i$, $\exp _{\text {it }}$ es una variable que capta la experiencia del individuo i en el año $t$, $u_{i t}$ es un término aleatorio y a, b y c son los valores que se estiman.

La variable educ puede ser cantidad de años de educación que posea el individuo o bien un conjunto de variables dummies que muestren el máximo nivel educativo que el individuo ha alcanzado. La variable exp puede ser la edad de la persona o una proxi de los años de permanencia en el mercado laboral (normalmente edad - años de educación $-6)$.

Si la variable educ es una variable continua (por ejemplo tiempo de educación, medido en años) se puede interpretar al coeficiente $b$ como la derivada parcial del ln $\mathrm{y}_{\mathrm{i}}$ con respecto a educ $\mathrm{c}_{\mathrm{i}}$. Bajo ciertos supuestos que se detallan más adelante, esta derivada parcial es el retorno a un año adicional de educación.

Una forma alternativa de calcular la rentabilidad del proyecto es calcular la tasa interna de retorno, resolviendo para $\mathrm{r}$ la siguiente ecuación:

$\mathrm{t}=\mathrm{E} \quad \mathrm{t}=\mathrm{T}$

${ }^{2}$ Ver Layard y Walters (1978), Cap 12. 


$$
\sum_{t=0}-\mathrm{Ct}(1+\mathrm{r})^{-\mathrm{t}}+\sum_{\mathrm{t}=\mathrm{E}+1} \mathrm{Rt}(1+\mathrm{r})^{-\mathrm{t}}=0
$$

Donde $\mathrm{Ct}$ representan los costos directos e indirectos y $\mathrm{Rt}$ son los diferenciales de salario de los que disfruta la persona más educada. E representa los años adicionales de educación, E+1 es el año de ingreso al mercado laboral y T es el año de retiro.

En realidad, el primer enfoque es un caso particular del segundo: se puede demostrar que el valor de $r$ que resuelve la ecuación 3, bajo ciertos supuestos, coincide con el valor estimado de b en la ecuación 2. Para ver esto, supongamos un individuo con E años de educación, que no trabaja durante sus años de estudios y entra al mercado laboral en el año $\mathrm{E}+1$, es decir, cuando finaliza sus estudios. Si el salario de esta persona es $\mathrm{y}_{\mathrm{E}}$, entonces el valor presente de los salarios que recibirá esta persona a lo largo de su vida es:

$\mathrm{VP}_{\mathrm{E}}=\mathrm{y}_{\mathrm{E}}(1+\mathrm{r})^{-(\mathrm{E}+1)}+\mathrm{y}_{\mathrm{E}}(1+\mathrm{r})^{-(\mathrm{E}+2)}+\ldots+\mathrm{y}_{\mathrm{E}}(1+\mathrm{r})^{-(\mathrm{E}+\mathrm{T})}$

En donde se supone que los salarios que recibe el trabajador se mantienen constantes en el tiempo, y el retiro se produce en el año T.

La ecuación 4 se puede reescribir como:

$\mathrm{VP}_{\mathrm{E}}=\mathrm{y}_{\mathrm{E}}(1+\mathrm{r})^{-\mathrm{E}}\left[1+(1+\mathrm{r})^{-1}+(1+\mathrm{r})^{-2}+\ldots+(1+\mathrm{r})^{-\mathrm{T}}\right]$

De forma similar, el valor presente de los ingresos de una persona con $E-j$ años de educación es:

$\mathrm{VP}_{\mathrm{E}-\mathrm{j}}=\mathrm{yE}_{\mathrm{E}-\mathrm{j}}(1+r)^{-(\mathrm{E}-\mathrm{j}+1)}+\mathrm{y}_{\mathrm{E}-\mathrm{j}}(1+r)^{-(\mathrm{E}-\mathrm{j}+2)}+\ldots+\mathrm{y}_{\mathrm{E}-\mathrm{j}}(1+r)^{-(\mathrm{E}-\mathrm{j}+\mathrm{T})}$

Que contiene los mismos supuestos que la ecuación 4, y se puede reescribir como:

$\mathrm{VP}_{\mathrm{E}-\mathrm{j}}=\mathrm{y}_{\mathrm{E}-\mathrm{j}}(1+\mathrm{r})^{-\mathrm{E}+\mathrm{j}}\left[1+(1+\mathrm{r})^{-1}+(1+\mathrm{r})^{-2}+\ldots+(1+\mathrm{r})^{-\mathrm{T}}\right]$

De acuerdo a la condición de equilibrio $1, \mathrm{VP}_{\mathrm{E}}=\mathrm{VP}_{\mathrm{E}-\mathrm{j}}$. Hay que notar que aquí se introduce, en forma implícita, otro supuesto: los únicos costos que acarrea la decisión de continuar los estudios son los salarios no percibidos, no hay costos directos, o son muy pequeños.

$\mathrm{Si} \mathrm{VP}_{\mathrm{E}}>\mathrm{VP}_{\mathrm{E}-\mathrm{j}}$ algunos individuos decidirían estudiar más, con lo que aumentaría la oferta relativa de personas educadas, caerían sus remuneraciones y los valores presentes tenderían a igualarse. Esto lleva a que a largo plazo,

$\mathrm{y}_{\mathrm{E}}(1+\mathrm{r})^{-\mathrm{E}}=\mathrm{y}_{\mathrm{E}-\mathrm{j}}(1+\mathrm{r})^{-\mathrm{E}+\mathrm{j}}$

Si suponemos que $E=j$, es decir, una persona sin educación, tenemos que

$\mathrm{y}_{\mathrm{E}}=(1+\mathrm{r})^{\mathrm{E}} \mathrm{y}_{0}$

Tomando logaritmos naturales en ambos lados de 9

$\ln \mathrm{y}_{\mathrm{E}}=\mathrm{E} \ln (1+\mathrm{r})+\ln \mathrm{y}_{0}$ 
en donde $b=\ln (1+r)$ que es aproximadamente $r$ para valores pequeños de $r$.

En resumen, el método para calcular los retornos a la educación por la función de Mincer (2) coincide con el método de la tasa interna de retorno (3), cuando se incorporan dos supuestos cruciales:

a) Los diferenciales de salarios entre trabajadores con distinto nivel educativo se mantienen constantes a lo largo del tiempo.

b) Los únicos costos de continuar los estudios son los salarios no percibidos durante ese período.

En este sentido, el método de la tasa interna de retorno es más general, ya que requiere menos supuestos. Los dos métodos, sin embargo, pueden ser estimados en forma estática o dinámica.

\subsubsection{El método estático}

La forma estática consiste en tomar los datos de un momento en el tiempo y construir las variables necesarias a partir de los datos de la muestra disponible. Es decir, se realiza un estudio de corte transversal.

Para armar los perfiles de ingreso - edad en un estudio de corte transversal se toman, para el momento de la muestra, los ingresos de las personas con distintas edades. Esto es, se observa a partir de los datos disponibles los ingresos de las personas de, digamos, 25 años, de 30 años, de 40 años, etc. con un cierto nivel educativo, y se supone que todas estas personas son una sola. De esta manera se obtiene el sendero de ingresos a lo largo del tiempo para un individuo con un cierto nivel educativo en el espacio ingreso edad. El mismo procedimiento se sigue para personas con otros niveles educativos y de esta forma se obtienen los ingresos que percibirán trabajadores con distinto grado de capacitación a lo largo de sus vidas. A partir de estos perfiles, se pueden estimar los retornos a la educación por cualquiera de los métodos enunciados.

La construcción de los perfiles de ingreso - edad a partir del procedimiento descripto en el párrafo anterior contiene un supuesto muy fuerte. Supone que los perfiles de ingreso edad para personas nacidas en diferentes momentos del tiempo se encuentran en la misma posición: supone que las personas de 25 años en el año $t$ con un nivel educativo $\mathrm{s}$ cuando tengan 30 años (en el año $\mathrm{t}+5$ ) van a percibir el mismo ingreso que las personas de 30 años con un nivel educativo s en el año $t$, $y$ que cuando tengan 40 años $(e n t+15)$ van a obtener los mismos ingresos que obtienen en $\mathrm{t}$ los individuos de 40 años con nivel educativo s, y así para todas las edades.

Esto no es necesariamente así en la realidad. Si por algún motivo los ingresos de un grupo de individuos aumenta o decrece en el tiempo (debido al crecimiento económico, cambio tecnológico, apertura, etc.) los perfiles de ingreso van a cambiar de posición. Como ejemplo, supongamos que la economía se encuentra en un proceso de crecimiento sostenido. Entonces, es probable que en el año $t+5$, las personas de 25 años en el año $t$ (de 30 años en $t+5$ ) disfruten de mayores ingresos que las personas de 30 años en el año 
t, debido al crecimiento de la economía. Lo mismo va a ocurrir cuando tengan 40 (en $\mathrm{t}+15$ ) con respecto a los de 40 años en $\mathrm{t}$ y para otras edades. Si los ingresos de la economía (o de un grupo particular de individuos) crecen a lo largo del tiempo, estimar los perfiles de ingreso - edad a través de la forma estática subestima los ingresos de ese grupo a lo largo de su vida. Lo contrario es válido si los ingresos de ese grupo decrecen a lo largo de los años: el perfil de ingreso - edad estático sobreestima los ingresos del grupo.

Esta idea se refleja con mayor claridad en el gráfico $\mathrm{N}^{\circ} 2$.

Gráfico $\mathrm{N}^{\circ}$ 2:

Perfiles de ingreso edad estáticos vs. dinámicos, para una generación imaginaria

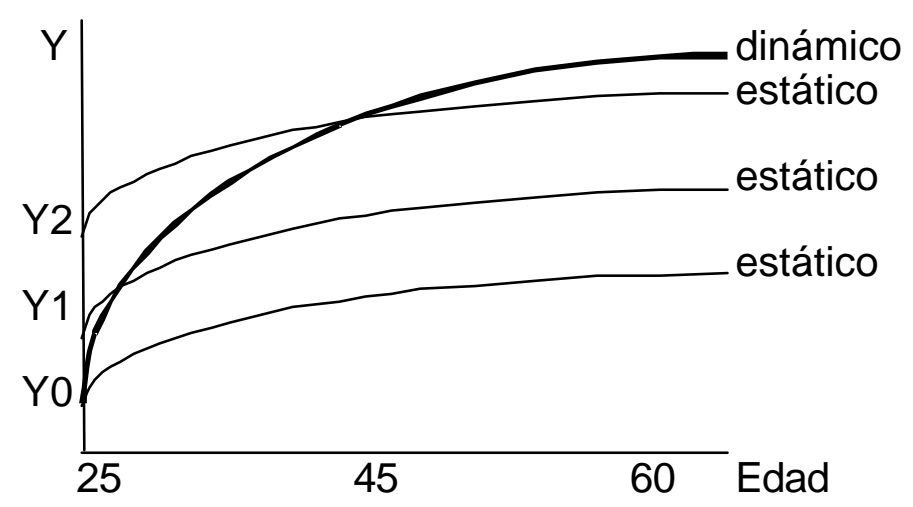

En el gráfico 2 se observa el inconveniente que se presenta cuando se estiman los perfiles de ingreso - edad para un grupo de individuos en forma estática. El contenido del gráfico es imaginario, pero sirve para ilustrar el problema.

Supongamos una generación como ejemplo, digamos, las personas nacidas en el año 1950 con secundaria completa. Supongamos además que la economía se encuentra en un proceso de crecimiento, por lo que los ingresos aumentan en el tiempo. Si se realizara un estudio estático en el año 1975, el perfil de ingreso - edad que se asignaría a este grupo sería la línea denominada estático 1975. El nivel de ingresos $\mathrm{Y}_{0}$ es el verdadero ingreso de este grupo, ya que en 1975 estas personas tienen 25 años. Pero el resto del sendero de ingresos no corresponde a esta generación. Se infiere a partir del ingreso de otros grupos. Como ejemplo: el ingreso de las personas de 40 años corresponde en realidad a los individuos nacidos en 1935 (que tienen 40 años en 1975, instante al que corresponden los datos), y el ingreso de las personas de 60 años corresponde al ingreso de los nacidos en 1915.

Si se realiza otro estudio de corte transversal en el año 1985, el perfil estimado sería el denominado estático 1985. Como la economía está en crecimiento, el ingreso de todas las personas aumenta y esto provoca que el perfil de 1985 se encuentre por encima del perfil para 1975. Ahora el nivel de ingresos $Y_{1}$ a la edad de 25 años corresponde a los nacidos en 1960, en tanto que el único punto de este sendero que pertenece al grupo investigado (la generación de 1950) es el de 35 años. Al igual que para el perfil anterior, los restantes puntos del perfil estático 1985 se infiren a partir de los ingresos de otras generaciones. Una situación similar se presenta para un estudio estático que se realice en 1995. El perfil de ingreso - edad se sitúa por encima del anterior debido al 
crecimiento, como ya se explicó. Ahora el único punto de este perfil que pertenece a los nacidos en 1950 es el que corresponde a la edad de 45 años.

\subsubsection{El método dinámico}

La base de datos ideal para analizar los fenómenos mencionados es una base de datos de panel, que permita seguir a individuos determinados a lo largo de sus vidas. Pero este tipo de base no se encuentra disponiblepara el caso argentino. Las encuestas de hogares son un panel rotativo, porque en cada muestra retienen al $75 \%$ de los hogares incluídos en la muestra anterior, y renuevan al $25 \%$ restante. Tienen elementos de un panel, porque se puede seguir a ciertos individuos en el tiempo, pero por la tasa de rotación sólo siguen a un hogar durante cuatro ondas. A los efectos de este estudio, se necesitaría un período mucho más largo. Sin embargo, las encuestas de hogares se llevan a cabo a intervalos regulares de tiempo, y aunque no permiten seguir a individuos durante lapsos prolongados, sí permiten seguir a grupos de individuos a lo largo de sus vidas. Como menciona Deaton (1997), un ejemplo obvio de grupo al cual se puede seguir a través del tiempo es el grupo de la población total. No es tan obvio cómo utilizar a las encuestas de hogares para seguir a cohortes a lo largo del tiempo, donde cohorte se define como un grupo de personas nacidas en un mismo año, y que reúnen ciertas características.

Si bien el analista no puede seguir a individuos determinados en el transcurso de sus vidas, si puede seguir a las medias, u otros estadísticos como por ejemplo percentiles de las variables de interés, de grupos determinados a lo largo de los años. En este sentido es como tener datos de panel no para individuos, sino para las medias de las variables de grupos de la población, en este caso, cohortes. Pero para realizar este seguimiento es necesario que ciertas características de los integrantes del grupo se mantengan a lo largo del tiempo. El objetivo es tener una población que mantenga sus características a partir de la cual se tome una muestra aleatoria. Y para esto es necesario que el grupo no se vea afectado por fenómenos migratorios, o que sus integrantes sean muy viejos y mueran en forma significativa, lo que puede reducir el número de integrantes a un nivel bajo, que no sea suficiente para obtener promedios confiables en términos estadísticos.

A diferencia del método estático, un estudio dinámico sigue al grupo bajo estudio a lo largo del tiempo. De esta forma, se tiene los ingresos de los nacidos en 1950 para el año 1975 (cuando este grupo tiene 25 años), para el años 1985 (35 años), 1995 (45 años), etc.

La ventaja que posee el método dinámico es que permite armar el perfil de ingreso edad para un determinado grupo estudiado a partir de los ingresos observados para ese grupo, en distintos momentos del tiempo, y no inferirlo a partir de los ingresos de otros grupos como ocurre en un estudio de corte transversal. Volviendo al ejemplo, el perfil de ingresos a partir de un estudio dinámico para la generación de 1950 computaría el ingreso de las personas de 25 años en 1975, de 35 años en 1985, de 45 años en 1995, y así para los años restantes. Siguiendo esta metodología, el perfil de ingresos que se obtiene es el denominado dinámico, que aparece en trazo grueso en el gráfico 2.

Para mostrar las consecuencias de emplear el método estático se usará el ejemplo hipotético citado. Si se tomara el estudio de 1975, el perfil de ingresos que se obtiene (estático 1975) subestima los verdaderos ingresos del grupo bajo estudio, ya que no incorpora el aumento del ingreso que obedece al crecimiento. Si se toma el estudio de 
1995, el perfil estático sobreestima los verdaderos ingresos, ya que incluye ingresos que no fueron percibidos por los nacidos en 1950, debido a que cuando eran jóvenes la economía se encontraba menos desarrollada y no tenían las mismas oportunidades que los jóvenes de 1995.

Si los ingresos del grupo bajo estudio decrecen a lo largo del tiempo, se obtiene la situación inversa. El estudio de 1975 sobrestimaría los verdaderos ingresos, y el de 1995 los subestimaría. Estas sobre o subestimaciones en los ingresos de un grupo de individuos tiene implicancias directas sobre el cálculo de las tasas de retorno. Si se subestiman los ingresos, se subestima el diferencial de salarios, y por lo tanto se hallará una tasa de retorno inferior a la verdadera. Si se sobrestiman los ingresos, se sobrestima el premio a la inversión, y la tasa de retorno que se asocia al proyecto es mayor que la real. esto no es un problema menor.

En la sección 4 se tratará de cuantificar estos problemas, para determinar en que medida los estudios estáticos sobre o subestiman las verdaderas tasas de retorno a la educación para la región del Gran Buenos Aires, entre los años 1974 y 1999.

\subsection{El método del trabajo: tasas de retorno dinámicas}

Como se señalara en la introducción, en este trabajo se emplea el método de la tasa interna de retorno para evaluar los retornos a la educación. Luego hay que comparar la tasa encontrada con la de mercado. Si es mayor, conviene realizar la inversión, ya que los fondos invertidos obtendrán una mayor rentabilidad que en un proyecto alternativo. Si es menor, significa que el costo de oportunidad de invertir los fondos en el proyecto educación no será compensado por los beneficios, y en consecuencia es preferible no llevar a cabo la inversión.

Esta forma de calcular los retornos a la educación presenta ventajas con respecto al cálculo estático a través de ecuaciones de Mincer para los ingresos. Primero, para interpretar los coeficientes de las funciones de Mincer (1974) como retornos a la educación, hay que suponer que el diferencial de ingresos de los individuos con mayor educación para una cohorte dada, es el mismo que obtuvieron las cohortes anteriores, y es el mismo que van a obtener las cohortes posteriores. Es decir, se supone que el diferencial de ingresos se mantiene constante a lo largo del tiempo (Arias y Mc Mahon, 1999). Esto es incorrecto cuando hay variaciones en los ingresos, ya sea que aumenten cuando el país crece, o que disminuyan por crisis macroeconómicas. Segundo, la ecuación de Mincer supone que los perfiles de ingreso-edad para las personas de un cierto nivel educativo son planos cuando se controla por la experiencia (Psacharopoulos, 1995). Lo que se observa son perfiles de ingreso-edad cóncavos, que parten de un ingreso mínimo, aumenta durante los primeros años de trabajo, alcanza un máximo y luego disminuyen hasta la edad del retiro. Tercero, se supone que los únicos costos incurridos para educarse son los ingresos no percibidos (los costos indirectos). En realidad también existen costos directos de educarse. En Argentina es probable que estos costos sean menores, comparados con los costos de oportunidad, pero en otros países no lo son. El cálculo por este método permite una mejor comparación con estudios realizados para otros países en donde los costos directos son importantes. Por último, para interpretar el coeficiente estimado de la ecuación de Mincer como el retorno a la educación, es necesario suponer que los costos anuales incurridos son aproximadamente iguales a los ingresos de una persona de similares características con 
un nivel inferior de educación, y que no existen costos directos. Si este no es el caso, hay que corregir el coeficiente estimado por un factor que refleje la relación entre los costos incurridos y los beneficios que se podrían obtener (ver Chiswick, 1997).

Los retornos que se calculan en el presente trabajo son retornos privados, debido a que se calculan tomando en cuenta los costos y beneficios privados. No se incluyen los costos para la sociedad de proveer educación (remuneraciones de los educadores, gastos en infraestructura, etc.) y los ingresos se toman antes de pagar impuestos, ya que no existe información disponible sobre ingresos después de impuestos.

El método elegido implica resolver la ecuación 3 para cada nivel educativo:

$$
\sum_{t=0}^{t=E}-C_{t}(1+r)^{-t}+\sum_{t=E+1}^{t=T}\left(R_{t}^{j}-R_{t}^{j-1}\right)(1+r)^{-t}=0
$$

El flujo de fondos se forma con los costos y beneficios de la decisión de continuar los estudios. Los costos se incurren durante los años de estudios, y están formados por la suma entre los salarios no percibidos, para cada año, y los costos directos. Los beneficios están formados por el diferencial de salarios del que disfruta el trabajador más capacitado, y se realizan a partir del momento mismo en que el estudiante se incorpora al mercado laboral. Se supone que esto ocurre cuando al finalizar el período de educación.

Para realizar los cálculos hay que tomar un nivel de educación como base. Como se explica más adelante, el nivel de educación mínimo incluido es primaria completa. Por lo tanto, se pueden extraer las tasas de retorno de los niveles superiores con respecto a la primaria completa, y entre otros niveles, por ejemplo, educación superior con respecto a educación secundaria, etc, pero no se puede calcular la tasa de retorno a la educación primaria, porque no hay un nivel inferior contra el cual comparar costos y beneficios. 


\section{Cuestiones empíricas}

Los datos utilizados para este trabajo provienen de la Encuesta Permanente de Hogares (EPH) que realiza el INDEC, para el Gran Buenos Aires, desde 1974 hasta 1999, período en el que las encuestas se han realizado a intervalos regulares. No se incluye a los aglomerados del interior del país porque no hay suficientes datos: las encuestas de hogares se encuentran disponibles a partir de 1992, y sólo para algunos aglomerados. Entonces, no se cuenta con suficientes años de observaciones, y se dificulta la comparación entre aglomerados.

\subsection{Población incluida}

En la muestra se va a incluir a individuos de sexo masculino, entre 12 y 60 años, de todos los niveles educativos, con respuestas completas, que formen parte del mercado laboral y tengan ingresos no negativos.

La edad de 60 años se establece como límite superior porque durante gran parte del período de estudio esa fue la edad a la que un trabajador se podía jubilar. En consecuencia, el número de individuos trabajadores mayores de 60 años es demasiado escaso como para obtener estimaciones confiables sobre ingreso promedio. Por otra parte, los ingresos jubilatorios se determinaban basándose en el sistema de reparto (lo que sigue ocurriendo actualmente para los trabajadores que no optaron por el sistema de capitalización), que es un sistema que tiende a igualar los ingresos de los jubilados con independencia de lo realizado por cada uno durante su vida laboral. No es que el sistema de reparto iguale todas las jubilaciones, pero sí tiene un componente igualador que distorsiona la información. Es muy probable que este componente genere que la jubilación no sea solamente un reflejo de las decisiones de educación en el pasado, sino que incluya otros factores. A los efectos de este trabajo, el ingreso jubilatorio no capta todo el premio por inversiones pasadas, y por lo tanto no se lo incluye.

El límite inferior de 12 años tiene otros motivos. Como se explicará más adelante, los ingresos que se tomarán en cuenta son los ingresos laborales. El premio a la inversión en educación son salarios más altos, que se disfrutan durante la vida laboral, y el costo está constituido por los salarios que dejan de percibirse mientras se estudia. Para calcular los costos de la inversión en educación secundaria, hay que computar los salarios que reciben las personas con educación primaria desde los 12 hasta los 18 años (edad en que se concurre a la secundaria). Si bien no es legal emplear a menores de 14 años, en los hechos esto pasa, y debe ser tenido en cuenta para computar los costos de la inversión. Por esa razón se incluye a las personas que reciben ingresos desde los 12 años.

La razón para incluir sólo hombres se encuentra muy justificada en la literatura. Numerosos trabajos justifican la separación por sexo debido a que las variables que son relevantes para tomar la decisión de ingresar al mercado laboral difieren para hombres y mujeres. 


\subsection{Ingresos}

Los ingresos incluidos son los provenientes del trabajo: remuneraciones salariales y bonificaciones ocasionales. No se incluye a los ingresos por jubilaciones, ya que el régimen de reparto vigente durante gran parte del período distorsiona la calidad de la información: tiende a igualar los ingresos de las personas, independientemente de la educación de cada una, por su diseño y forma de funcionamiento. Sería interesante incluir a los ingresos provenientes de la propiedad, pero hay que estudiar cuidadosamente la calidad de estos ingresos en la EPH.

Debido a las crisis macroeconómicas del período analizado, se ajustaron los valores por las variaciones en el nivel de precios, según el IPC, y se tuvo en cuenta el cambio de unidad monetaria de enero de 1992. Es conocido que existe subdeclaración en los ingresos, sobre todo para los deciles superiores. En este trabajo los ingresos no son ajustados por subdeclaración, por dos razones. Primero, se toman en cuenta sólo los ingresos laborales, que reciben el mismo factor de ajuste por subdeclaración, con independencia de los deciles, por lo que no se alteran los ingresos relativos de los distintos grupos educativos. En consecuencia, los resultados no cambian cuando se realiza el ajuste. Segundo, el cálculo de los factores de ajuste por subdeclaración tiene ciertos problemas metodológicos, ya que compara los ingresos de las encuestas de hogares con los de la cuentas nacionales, que se toman en forma distinta (Gasparini y Sosa Escudero 2000). Para evitar incorporar estos problemas metodológicos, y debido a que los resultados no se modificarían en caso de realizarse el ajuste, los ingresos no se ajustan por subdeclaración.

\subsection{Niveles educativos}

La EPH define cinco niveles y dos categorías educativas. Los niveles son: personas que nunca han concurrido a la escuela, educación primaria, educación secundaria, educación terciaria y educación universitaria. Las categorías se refieren a si los niveles han sido completados o no. Para calcular las tasas de retorno voy a incluir cinco niveles: primaria completa (7 años de educación, si no se computa el nivel preescolar), secundaria incompleta (entre 8 y 11 años), secundaria completa (12 años), educación superior incompleta, que incluye a los niveles terciario y universitario, (entre 13 y 16 años) y educación superior completa (17 años). No se incluyen a las personas que nunca concurrieron a la escuela, ni a los que no completaron el nivel primario, porque para la región y período considerados representan un muy bajo porcentaje del total de la muestra.

\subsection{Las cohortes}

En este trabajo, una cohorte se define como un grupo de individuos del mismo sexo, nacidos durante un período de tiempo. Las observaciones de la muestra serán divididas en "celdas", en donde cada una contiene a los individuos hombres nacidos en el mismo período, que han alcanzado el mismo nivel educativo, y hayan declarado ingresos no negativos. El dato de cada celda es un promedio de los individuos incluidos en ella.

En el armado de las cohortes surgieron cuatro cuestiones a resolver: 
1) Debido a que las cohortes se definen por la edad de los individuos, se trabaja sólo con las ondas del mes de octubre. Incluir las ondas de mayo agrega observaciones, pero introduce dificultades insalvables, ya que los individuos que nacieron entre mayo y octubre aparecen con edades diferentes en cada onda. Esto genera que de mayo a octubre cambien de cohorte. Individuos que en mayo aparecerían como dentro de la cohorte de, digamos, 25 años, aparecerán en octubre agrupados con los individuos de 26 años, entre los que se encuentran los que tenían 26 años en mayo. De esta forma los individuos que pertenecen a un grupo se mezclan con los de otro grupo diferente, y el límite entre las cohortes se vuelve borroso.

2) Las cohortes, cuando hay datos suficientes, agrupan a los individuos nacidos en el mismo año. En este trabajo, la cohorte será una observación El dato es un promedio de los integrantes de esa cohorte. Para tener un dato confiable, es necesario tener muchos integrantes. Para ciertos niveles educativos (sobre todo nivel superior completo) si se toma un año solo para definir la cohorte se tienen pocos individuos. Para contar con grupos más numerosos, se toman bandas de cinco años para definir las cohortes.

3) El nivel superior incompleto tenía muy pocos integrantes aún tomando bandas de cinco años. Como los ingresos de este grupo no diferían estadísticamente de los ingresos de las personas con nivel secundario completo, los niveles superior incompleto y secundaria completo aparecen juntos bajo la denominación secundario completo.

4) La vida laboral de un individuo normalmente es de 40 años aproximadamente. Para armar el perfil de ingresos a lo largo de su vida, se necesitan entonces 40 años, a los que hay que sumar los años de educación. Los datos de las encuestas se encuentran disponibles desde 1974, lo que totaliza 25 años. Para las cohortes jóvenes, no se cuenta con información sobre los ingresos de sus últimos años de vida laboral. Para completar los años que no se observan y poder obtener una estimación del retorno dinámico, se dividieron a las cohortes en distintos grupos (por niveles educativos) y se ajustaron los ingresos cada grupo por las tasas de crecimiento que se registraron para grupos similares de cohortes más viejas.

Supongamos que una cohorte A se observa hasta los 40 años. Para completar los ingresos, se ajusta el ingreso de cada grupo perteneciente a la cohorte A a los 40 años por las tasas de crecimiento de los ingresos de los grupos correspondientes a una cohorte B (que si se observan) para las edades que faltan, en este caso, de los 41 hasta los 60 años. Se toma la tasa de crecimiento de los ingresos de los grupos de la cohorte B de los 41 hasta los 50 años y se le aplica ese porcentaje al ingreso de cada grupo de de la cohorte A para obtener los ingresos desde los 41 hasta los 50 años. Luego se toma la tasa de crecimiento promedio de los ingresos para cada grupo de la cohorte B (u otra a la que se le puedan observar los ingresos) desde los 51 hasta los 60 años y se le aplica ese porcentaje a los ingreso de los grupos de la cohorte $\mathrm{A}$, hasta obtener los ingresos de toda la vida laboral.

En este trabajo se ha realizado una sola modificación a este procedimiento: en vez de aplicar las tasas de crecimiento de los salarios de otros grupos al último salario observado, el de 1999, se han aplicado las tasas de crecimiento al salario promedio de los últimos cinco años para cada grupo educativo. La razón del cambio obedece a que los ingresos de 1999 son bastante más bajos que el promedio para los últimos cinco o diez años, por la crisis financiera que se vivió en el país. Entonces, aplicar las tasas a un 
salario inicial extraordinariamente bajo, puede sesgar hacia abajo todos los años restantes, distorsionando las estimaciones.

Para las cohortes más viejas, lo que no se observa son los ingresos durante los primero años de su vida. En este caso se siguió el procedimiento descripto: se tomaron las tasas de crecimiento de los ingresos de las cohortes jóvenes en los primeros años de trabajo, y se aplicaron a los ingresos de las cohortes mayores.

Este procedimiento es el más utilizado en la literatura (Rosen 1977 y Arias y Mac Mahon 1999) y se ha verificado, con datos ex-post, que las tasas dinámicas no se ven muy afectadas. Si bien tiene ciertas características asociadas al método estático, ya que se toma información de individuos de otras edades para completar los ingresos de un grupo, no es exactamente igual, debido a que se parte de los ingresos del grupo que se quiere analizar, y por lo tanto se permiten diferencias en la posición de los perfiles de ingreso - edad, que es la pricipal ventaja del método dinámico.

\section{Tasas de retorno para el Gran Buenos Aires, 1974 - 1999}

\subsection{Evolución de los ingresos laborales, GBA 1974 - 1999}

En la tabla $\mathrm{N}^{\circ} 1$ se definen las variables del trabajo. En la tabla $\mathrm{N}^{\circ} 2$ se muestra la evolución de los ingresos laborales para el aglomerado del Gran Buenos Aires, desde 1974 hasta 1999. El período de estudio se divide en dos subperíodos en los cuales la economía Argentina ha estado bajo distintos regímenes macroeconómicos.

Tabla 1. Definición de variables

\begin{tabular}{|c|c|}
\hline Variable & Definición \\
\hline \multicolumn{2}{|c|}{ 1. Educación } \\
\hline epi & Educación primaria incompleta (4 años de educación) \\
\hline epc & Educación primaria completa (7 años de educación) \\
\hline esi & Educación secundaria incompleta (10 años de educación) \\
\hline esc & $\begin{array}{l}\text { Educación secundaria completa. Incluye a las personas que tienen } \\
\text { educación superior incompleta ( } 12 \text { o } 15 \text { años de educación) }\end{array}$ \\
\hline euc & $\begin{array}{l}\text { Educación superior (universitaria o terciaria) completa (17 años de } \\
\text { educación) }\end{array}$ \\
\hline \multicolumn{2}{|l|}{ 2. Cohortes } \\
\hline Cohorte 4 & Hombres nacidos entre 1938 y 1942 \\
\hline Cohorte 5 & Hombres nacidos entre 1943 y 1947 \\
\hline Cohorte 6 & Hombres nacidos entre 1948 y 1952 \\
\hline Cohorte 7 & Hombres nacidos entre 1953 y 1957 \\
\hline Cohorte 8 & Hombres nacidos entre 1958 y 1962 \\
\hline Cohorte 9 & Hombres nacidos entre 1963 y 1967 \\
\hline Cohorte 10 & Hombres nacidos entre 1968 y 1972 \\
\hline
\end{tabular}

La tabla 2a muestra la evolución de los ingresos laborales por niveles educativos, incluyendo a los individuos de todas las edades. En ella se observa que para todo el período los ingresos de todos los niveles han experimentado una variación negativa, $\mathrm{y}$ que los ingresos de los grupos con menor educación han caído a una tasa mayor. A mayor nivel educativo, menor es la caída en los salarios reales. Pero si observamos los 
dos subperíodos se puede ver que el comportamiento de los ingresos ha sido distinto. Desde 1974 hasta 1990 el ingreso cae para todos los grupos, y disminuye menos para los niveles con mayor educación, aunque las personas con secundaria completa experimentan una disminución menor que las personas con nivel universitario. Los niveles más perjudicados son primaria incompleta y secundaria incompleta. Los cambios que se observan a partir de 1991 y hasta 1999 son diferentes. Los ingresos de todos los grupos aumentan en la primera parte de la década, pero hacia el final experimenttan un descenso mayor, por lo que para el período se observa en promedio una baja en los ingresos de todos los grupo, con excepción del universitario. Las mayores caídas las sufren los niveles secundario completo y primario completo, a diferencia del período anterior.

En la tabla $2 b$ se muestra la evolución de los ingresos por cohortes, sin distinguir entre niveles educativos. En general se observa que las cohortes más jóvenes están en mejor posición relativa, ya sea porque sus ingresos disminuyen menos o aumentan en mayor proporción que los de las cohortes más viejas. Este hecho puede deberse a que las cohortes más viejas se encuentran en sus últimos años de vida laboral, en los que los ingresos suelen ser menores. En cambio las cohortes más jóvenes se encuentran en una etapa de su vida en donde sus ingresos crecen, porque ganan en experiencia o encuentran el trabajo para los que son más productivos. El caso de la cohorte 10 merece una mención: este grupo está formado por los individuos nacidos entre 1968 y 1972 e ingresa al mercado laboral en la década del 90, por lo tanto capta la suba de los ingresos de este período, sin sufrir la crisis de la década anterior, debido a que en esos años se encontraban aun dentro del sistema educativo. Esa es la causa del crecimiento tan dispar respecto de las otras cohortes.

\section{Tabla $\mathrm{N}^{\circ} 2$}

Tasas anuales promedio de crecimiento de los salarios reales. Gran Buenos Aires, 1974-1999, en pesos de oct. 1998

2a. Por niveles educativos, todas las cohortes

\begin{tabular}{lccc}
\hline & \multicolumn{3}{c}{ Variaciones anuales promedio } \\
\hline & $1974-99$ & $1974-91$ & $1991-99$ \\
\hline epi & $-2.9 \%$ & $-3.6 \%$ & $-1.6 \%$ \\
epc & $-2.7 \%$ & $-2.8 \%$ & $-2.4 \%$ \\
esi & $-2.7 \%$ & $-3.7 \%$ & $-0.7 \%$ \\
esc & $-2.3 \%$ & $-1.9 \%$ & $-3.2 \%$ \\
euc & $-1.5 \%$ & $-2.6 \%$ & $0.9 \%$ \\
\hline \hline
\end{tabular}


2b. Por cohortes, todos los niveles educativos

\begin{tabular}{lccc}
\hline & \multicolumn{3}{c}{ Variaciones anuales promedio } \\
\hline Grupo & $1974-99$ & $1974-91$ & $1991-99$ \\
\hline Cohorte 4 & $-2.0 \%$ & $-1.8 \%$ & $-2.1 \%$ \\
Cohorte 5 & $-1.9 \%$ & $-1.3 \%$ & $-2.7 \%$ \\
Cohorte 6 & $-1.0 \%$ & $-2.2 \%$ & $1.1 \%$ \\
Cohorte 7 & $-0.4 \%$ & $-0.5 \%$ & $-0.3 \%$ \\
Cohorte 8 & $-0.6 \%$ & $-1.4 \%$ & $0.0 \%$ \\
Cohorte 9 & $0.9 \%$ & $0.5 \%$ & $1.2 \%$ \\
Cohorte 10 & $2.9 \%$ & & $2.9 \%$ \\
\hline \hline
\end{tabular}

Con la información de ingresos, se puede proceder a estimar los costos y beneficios de la inversión en capital humano.

\subsection{La estimación de los costos y beneficios}

Los costos del proyecto de inversión en educación son de dos tipos. Los costos directos están integrados por las erogaciones necesarias para realizar el aprendizaje, y están formados por la compra de textos y otros materiales necesarios para cumplir con los requisitos de los estudios. También incluyen los montos pagados por el estudiante, si la educación no es gratuita. Los costos indirectos representan el costo de oportunidad, los salarios que se podrían obtener en el mercado laboral, para personas con las mismas características del estudiante, pero que no se perciben porque la persona estudia todo el tiempo.

Para el período de estudio no se cuenta con información sobre los costos directos. Se pueden calcular a partir de las encuestas de gastos, pero éstas son muy recientes y sus resultados difícilmente puedan usarse para estimar los costos directos de las décadas del 70 y 80 . Además, debido a que la educación en Argentina es gratuita ${ }^{3}$ se puede suponer que los costos directos tienen una participación muy pequeña en los costos totales. Por estos motivos se realiza el supuesto de que los costos directos son iguales a cero, y los únicos costos de continuar estudiando son los costos indirectos.

Los costos indirectos son los costos de oportunidad asociados al proyecto, constituidos por el valor monetario del tiempo invertido en estudiar. Se miden por el ingreso que recibe una persona con las mismas características del estudiante (sexo, edad, etc.) pero que no estudia y dedica su tiempo a trabajar. En la ecuación 12, $\mathrm{C}_{t}$ es el salario promedio del grupo de trabajadores del mismo sexo y de la misma cohorte que el grupo estudiado, pero con un nivel educativo inferior.

Los beneficios de la inversión en educación están compuestos por los mayores ingresos que percibe, a lo largo de su vida laboral, un trabajador más capacitado. Para estimarlos se toma la diferencia entre los ingresos promedio de dos grupos que tiene el mismo sexo $\mathrm{y}$ forman parte de la misma cohorte, pero que difieren en el nivel educativo alcanzado. En la ecuación $12, \mathrm{R}_{\mathrm{t}}^{\mathrm{j}}$ es el ingreso promedio de los individuos de una cohorte en el año

\footnotetext{
${ }^{3}$ Si bien existen establecimientos privados que cobran una matrícula, siempre existe la opción de concurrir a un establecimiento del Estado, que brinda los servicios en forma gratuita.
} 
$\mathrm{t}$ con nivel educativo $\mathrm{j}$, $\mathrm{y} \mathrm{R}^{\mathrm{j}-1}$ es el ingreso promedio de los individuos de la misma cohorte en el año t con el nivel educativo inmediato inferior.

\subsection{Tasas de retorno estáticas}

En la tabla 3 se muestran las estimaciones de los retornos a la educación por el método TIR estático descripto en la sección 2.2.1. Los rendimientos del nivel primaria completa no se estiman ya que no hay suficiente información para calcular los costos de invertir en este nivel (serían los salarios de trabajadores con primaria incompleta, que son escasos para obtener estimaciones confiables). Para calcular estos retornos se hizo un estudio por año, es decir, 17 estimaciones usando datos de corte transversal. Para cada año y nivel eduactivo, se obtiene el ingreso medio de los individuos para cada edad, desde los 18 (24 en el caso de educación superior) hasta los 59 años. De esta forma se arman los perfiles de ingresos, se computan los costos de oportunidad y se obtienen los diferenciales de salarios por nivel educativo. Luego se estiman los retornos empleando el método TIR estático.

Tabla 3. Tasas de retorno estáticas. Gran Buenos Aires, 1980 - 1999.

\begin{tabular}{cccc}
\hline Año & \multicolumn{3}{c}{ Nivel educativo } \\
\hline & Esi & Esc & Euc \\
\hline \hline 1980 & $11 \%$ & $14 \%$ & $19 \%$ \\
1982 & $14 \%$ & $14 \%$ & $18 \%$ \\
1985 & $10 \%$ & $14 \%$ & $14 \%$ \\
1986 & $13 \%$ & $12 \%$ & $17 \%$ \\
1987 & $11 \%$ & $13 \%$ & $18 \%$ \\
1988 & $13 \%$ & $18 \%$ & $15 \%$ \\
1989 & $13 \%$ & $16 \%$ & $17 \%$ \\
1990 & $14 \%$ & $13 \%$ & $16 \%$ \\
1991 & $11 \%$ & $13 \%$ & $19 \%$ \\
1992 & $11 \%$ & $11 \%$ & $18 \%$ \\
1993 & $16 \%$ & $12 \%$ & $20 \%$ \\
1994 & $12 \%$ & $11 \%$ & $21 \%$ \\
1995 & $9 \%$ & $13 \%$ & $22 \%$ \\
1996 & $10 \%$ & $11 \%$ & $22 \%$ \\
1997 & $14 \%$ & $11 \%$ & $23 \%$ \\
1998 & $13 \%$ & $14 \%$ & $24 \%$ \\
1999 & $10 \%$ & $11 \%$ & $23 \%$ \\
\hline Promedio & $12.0 \%$ & $13.0 \%$ & $19.1 \%$ \\
\hline \hline
\end{tabular}

Aquí hay que resaltar un hecho. Existe una gran variabilidad de los retornos dentro de cada grupo. Para el caso de las personas con educación secundaria incompleta, los retornos van del $9 \%$ al 16\%, para el secundario completo del $11 \%$ al $18 \%$ y para el nivel superior completo del $14 \%$ al $24 \%$. Cuando hablamos de tasas de retorno, diferencias de 7 o 10 puntos porcentuales son muy significativas. Esto obedece a que los ingresos se ven afectados por shocks de corto plazo, que se trasladan totalmente a los retornos, por la forma en que se calculan: los cambios en los ingresos relativos de los grupos en un año determinado se trasladan totalmente a los retornos estimados para ese año. Como ejemplo de esto puede mencionarse que los retornos anuales para el nivel secundario incompleto son en promedio 2,5\% menores a los del nivel secundario completo para la 
década del 80, en tanto que no hay diferencias para la década del 90. Esto no quiere decir que el retorno a la educación secundaria completa sea cero, porque los retornos se calculan con respecto al nivel anterior. La interpretación correcta es que el retorno del nivel secundario incompleto, respecto del nivel primario completo, coincide con el retorno del nivel secundario completo, respecto del secundario incompleto. La explicación de esto radica en el cambio de los ingresos que experimentaron los dos grupos. En la década del 80, disminuyeron en menor medida para el secundario completo, tendencia que se revierte en la década del 90. Algo similar ocurre con los universitarios. En la década del 90, cuando sus ingresos aumentan, las tasas de retorno de este grupo aumentan, tanto en términos relativos como absolutos, llegando a superar el $20 \%$ anual.

El punto central, volviendo al ejemplo de los niveles secundarios, es que de acuerdo a los retornos estáticos, se puede decir que para la década del 80 los retornos a la educación son crecientes (ambos niveles presentan tasas positivas) y a tasas crecientes (la tasa para el nivel esc es mayor que para el nivel esi), mientras que para la década del 90 se puede decir que los retornos son crecientes, a tasa constante (las tasas para los dos niveles coinciden en promedio). En realidad, una persona con secundaria completa que trabaje en las dos décadas observará que su diferencial de salarios es mayor durante los primeros diez años y se reduce luego. De esta forma, es probable que los efectos se compensen, y no se pueda establecer con claridad si los retornos crecen a tasa constante, creciente o decreciente. Para determinar cual es el caso, hay que utilizar las tasas dinámicas, que observan los diferenciales de ingresos de un trabajador a lo largo de toda su vida, y no lo infieren a partir del ingreso de otros grupos de personas.

En el estudio citado de Pessino (1995) se calculan tasas estáticas. De acuerdo a los resultados que se presentan, las tasas de retorno (por nivel educativo) aumentan con la hiperinflación de 1989 y luego se estabilizan en 1993, en niveles comparables a los de 1986. De estos resultados se concluye que la distribución del ingreso empeora con la inflación, ya que provoca un aumento en los retornos a la educación, y se supone una relación positiva entre estos retornos y la desigualdad en la distribución del ingreso. Una limitación del trabajo (reconocida por la autora) es que no pueden separar los efectos que tienen sobre la distribución del ingreso los cambios en los retornos a la educación y el shock macroeconómico. Entonces, se atribuye todo el efecto a la educación. Otra limitación, generada por el uso del método estático, es que probablemente se sobrestimen (tanto hacia arriba como hacia abajo) los cambios en los retornos a la educación. Es cierto que un shock que afecte los ingresos provoca un cambio en los retornos, pero si el shock desaparece y los ingresos se estabilizan (hecho que se confirma en Pessino, y en este trabajo, ver tabla 2) entonces el cambio en los retornos es sólo de corto plazo. En términos de los retornos dinámicos, el shock se diluye en el tiempo, y el efecto de largo plazo es menor al impacto de corto plazo.

Teniendo en cuenta las observaciones anteriores, de acuerdo a este método, en promedio para todo el período, los retornos a la educación aumentan con el nivel educativo y a tasa creciente. De los resultados anteriores no se pueden derivarse conclusiones en cuanto a la evolución de los retornos a la educación en el tiempo, debido a las debilidades que presenta el método estático, mencionadas en la sección 2.2.1. Los resultados se muestran a los efectos de poder compararlos con las estimaciones dinámicas. 


\subsection{Tasas de retorno dinámicas}

En esta sección se presentan los resultados de las estimaciones de la tasas de retorno por nivel educativo por el método dinámico descripto en el punto 4. Este método utiliza los ingresos observados de un grupo de individuos a lo largo de sus vidas. Debido a que no se dispone de encuestas durante un período lo suficientemente largo como para observar todo el perfil de ingresos de todos los grupos, se completaron los años no observados empleando el procedimiento mencionado en el punto 3.4.

Tabla $N^{\circ}$ 5. Tasas de retorno dinámicas, GBA 1974 - 1999.

\begin{tabular}{ccccc}
\hline Cohorte & \multicolumn{4}{c}{ Nivel educativo } \\
\hline & epc & esi & esc & euc \\
\hline \hline Cohorte 4 & $14.2 \%$ & $9.2 \%$ & $10.4 \%$ & $13.8 \%$ \\
Cohorte 5 & $14.5 \%$ & $11.0 \%$ & $10.5 \%$ & $14.3 \%$ \\
Cohorte 6 & $10.3 \%$ & $13.4 \%$ & $10.6 \%$ & $11.3 \%$ \\
Cohorte 7 & $6.1 \%$ & $9.0 \%$ & $6.3 \%$ & $10.6 \%$ \\
Cohorte 8 & $6.1 \%$ & $7.8 \%$ & $6.8 \%$ & $11.5 \%$ \\
Cohorte 9 & $8.9 \%$ & $6.3 \%$ & $5.6 \%$ & $16.8 \%$ \\
Cohorte 10 & $9.3 \%$ & $9.5 \%$ & $8.4 \%$ & $22.0 \%$ \\
\hline Promedio & $9.9 \%$ & $9.4 \%$ & $8.4 \%$ & $14.3 \%$ \\
\hline \hline
\end{tabular}

Las tasas de retorno de la tabla muestran la TIR del flujo de fondos asociado al proyecto de continuar estudiando. Esto es, cuál es el redimiento anual de continuar los estudios, respecto del nivel inmediato anterior, para un individuo promedio: que finalice sus estudios secundarios o universitarios en cinco años, o en la mitad del tiempo si es que no completa el nivel, que no trabaje mientras estudia, en donde los únicos costos por estudiar son los costos indirectos (de oportunidad), formados por los salarios de individuos con idénticas características con excepción del nivel educativo alcanzado y que trabaje durante desde que finaliza sus estudios hasta los 60 años. Es decir que un individuo promedio perteneciente a la cohorte 4 que tenga estudios secundarios completos, obtendrá un retorno del $10,4 \%$ anual por cada peso invertido en su educación secundaria.

Los resultados obtenidos pueden ser interpretados como el retorno a alcanzar un determinado nivel educativo, ya que los ingresos y costos utilizados para la estimación son los observados para un grupo a lo largo de su vida, y no se infieren a partir de los ingresos de otros grupos, como en el método estático, lo que obliga a exigir diferenciales de ingreso constantes entre grupos para poder interpretar el coeficiente estimado como retorno.

Estas tasas de retorno no deben interpretarse como el rendimiento de un año adicional de educación, ya que no se definen por años de estudios sino por nivel alcanzado. En este sentido no puede hablarse estrictamente de tasas marginales por nivel educativo, ya que los niveles educativos no son una variable continua, sino discreta. Representan el retorno anual, en pesos, de alcanzar un determinado nivel educativo.

\subsubsection{Las tasas de retorno dinámicas por cohorte y nivel educativo}


Si comparamos los resultados promedio (para todas las cohortes) por nivel educativo, se observa que la educación tiene rendimientos positivos para todos los niveles. En cuanto al retorno incremental (ya que no se puede hablar de retorno marginal), es decreciente para el nivel secundario completo, y creciente para el nivel universitario completo. El análisis de las causas de este resultado excede al objetivo de este trabajo, pero se puede pensar que están relacionados con la oferta y la demanda de trabajo calificado y no calificado: el nivel educativo promedio de la población ha aumentado en forma sistemática durante el período de estudio. De 1980 a 1999, los hombres que trabajan con secundario incompleto han pasado del $21,8 \%$ al $27 \%$ de todos los trabajadores, los hombres con secundario completo del $17,9 \%$ al $26,4 \%$ y los hombres con estudios universitarios completos del 3,8\% al 8,7\%. Es decir, se verifica un aumento en el nivel educativo de la fuerza de trabajo empleada. Pero el mayor incremento en la cantidad lo tienen los universitarios, que más que duplican su participación. Si a esto le agregamos que durante la década del 90 es el único grupo que experimenta crecimiento en sus ingresos reales, se puede pensar que la demanda relativa de trabajo calificado ha aumentado y como la oferta de esta clase de trabajo tarda en adaptarse a los cambios en la demanda, el precio relativo aumenta (García Swartz 1996), lo que se refleja en diferenciales de ingresos, y por lo tanto en retornos, más elevados.

El análisis entre cohortes hay que dividirlo por niveles educativos. Para los niveles secundarios, las cohortes más viejas en general tienen retornos mayores que las cohortes jóvenes. Esto puede deberse a la caída constante en los salarios reales. El caso de la cohorte 10 será analizado más adelante, en conjunto con el nivel universitario. Cuando se mira este nivel, ocurre lo mismo para las cohortes 4 a 8 , pero la 9 y la 10 , sobre todo la última, tienen retornos relativamente muy altos. La causa es que los integrantes de estas cohortes entran al mercado laboral en la década del 90, y disfrutan desde el inicio del aumento de ingresos de la primera etapa de este decenio. Además, incurrieron sus costos en la última etapa de la década del 80, con ingresos (y por lo tanto costos de oportunidad) relativamente más bajos que los ingresos (beneficios) de los años siguientes. En el caso de los universitarios, este efecto se agranda ya que los salarios reales de este grupo crecen a una tasa positiva desde 1991. Estos efectos explican por qué la cohorte 10 tiene rendimientos más altos que las cohortes 7,8 y 9 , y el extraordinario $22 \%$ de los universitarios. De todos modos, es probable que los ingresos de este cohorte a partir del cual se armaron los diferenciales para el resto de los años no observados, sean muy variables, por lo que las estimaciones para esta cohorte tiene que ser analizada con precaución. Excepto para la cohorte 4, se mantiene el fenómeno observado para el promedio: el retorno para el nivel secundario incompleto es mayor que el retorno para el secundario completo, que a su vez es menor que el retono para el nivel universitario.

Los retornos para el nivel primario completo se presentan con fines ilustrativos. No son resultados que se puedan interpretar con el mismo grado de confianza que el resto. El problema es que para determinar estos retornos, es necesario conocer los ingresos de los trabajadores con primario incompleto, desde los 9 o 10 años hasta los 13 años, con el fin de determinar los costos de oportunidad (los ingresos no percibidos) de terminar el nivel primario. Pero el número de observaciones es muy reducido, en general sólo 2 a 5 por encuesta, y por lo tanto el ingreso usado como costo de oportunidad es un promedio obtenido a partir de muy pocas observaciones y en consecuencia poco confiable. Por este motivo los resultados sólo se muestran pero no se analizan. 


\subsubsection{Comparaciones con las tasas de retorno estáticas}

Si comparamos las tasas de retorno estáticas (en promedio para todo el período) con las tasas dinámicas (en promedio para todas las cohortes) encontramos que las primeras son superiores a las segundas. Las tasas estáticas para los niveles secundario incompleto, secundario completo y universitario son $12,0 \%, 13,0 \%$ y $19,1 \%$ respectivamente. Las tasas dinámicas, manteniendo el orden, son 9,4\%, 8,4\% y 14,3\%. Es decir, que los retornos estáticos sobreestiman a los dinámicos en 2,6\% para el nivel secundario incompleto, 4,6\% para el nivel secundario incompleto y 4,8\% para el nivel universitario completo.

Se puede ver aquí el problema de estimar los retornos a la educación por el método estático. En una economía cuyos ingresos reales caen en forma permanente, los resultados del método estático sobreestiman los verdaderos retornos, que se pueden obtener por el método dinámico.Es la consecuencia establecida en la sección 2.2. La causa radica en que cuando se infieren los ingresos de determinado grupo a partir de los ingresos de otros grupos, se supone implícitamente que el nivel de ingresos se mantiene entre grupos. Cuando los ingresos de toda la economía, o de un grupo particular, disminuyen a lo largo del tiempo, es muy probale que los ingresos inferidos para realizar el cálculo por el método estático sean mayores a los que se "realizan", a los que terminan observándose en realidad. En consecuencia, el método estático genera un perfil de ingresos mayor al real, y por lo tanto el retorno se sobreestima.

\subsubsection{Limitaciones de las estimaciones}

El análisis, a pesar de ser más apropiado en un sentido que el método estático, tiene sus limitaciones. En general son de orden práctico, y tiene que ver con la información disponible y la técnica empleada para obtener los datos de las encuestas.

En primer lugar, y como se mencionó anteriormente, las encuestas están disponibles para un período relativamente largo, pero no suficiente para abarcar todos los años de estudio y trabajo de las cohortes. Por lo tanto es necesario completar los datos faltantes realizando inferencias a partir de información obtenida de otras cohortes. Si bien el procedimiento empleado no tiene tantas limitaciones como el método estático, sería mejor contar con todos los datos para una cohorte.

Segundo, se supone que los estudiantes no trabajan, y se terminan sus estudios en el período estipulado en los programas de cada nivel, o en la mitad del tiempo si es que los abandonan. En las encuestas se puede determinar si un estudiante trabaja, pero no se puede determinar en cuanto se alarga el período de estudio (si es que en realidad se alarga, como es esperable). Para un estudiante que trabaje, los costos de oportunidad son menores, pero se incurren durante un mayor número de años (también se trabaja tiempo completo durante menos años) con lo que hay efectos que juegan en sentidos opuestos a los efectos de calcular el retorno. Como la información que brinda la EPH no permite distinguir estos efectos, se supone que los estudiantes no trabajan. Tampoco es posible obtener los años promedio de estudios para los estudiantes que no finalizan un determinado nivel, por lo que el dato ha sido obtenido a partir de una fuente externa (Llach y otros 1999). 
Tercero, existen los problemas de autoselección y el grupo contrafactual. El problema de la autoselección se origina en que probablemente los individuos con mayor habilidad se "autoseleccionen" para continuar los estudios. Las personas más hábiles saben que son más aptas para los trabajos que requieren mayor capacitación, y endógenamente deciden invertir en capital humano. En este sentido, la muestra no es aleatoria. La intuición es que el retorno a la educación, neto de este efecto de habilidad, debería ser menor, pero numerosos estudios sobre el tema no han llegado a ningún resultado definitivo. Con la información disponible para Argentina, no es posible abordar este tema. El problema del grupo contrafactual es que al investigador le gustaría comparar los ingresos de una persona, luego de haber invertido en un cierto nivel eduactivo, con los ingresos de la misma persona, si no hubiera realizado la inversión. Como esto es imposible, se realiza la comparación contra un grupo de personas similares, pero que no son las mismas, y por lo tanto puede existir diferencias inobservables para el investigador.

Por último, y muy importante. Durante todo el análisis, se supuso que la decisión de estudiar o no se tomaba en base a variables exclusivamente monetarias. La única recompensa por invertir en educación son los mayores ingresos monetarios que se perciben respecto del trabajador con menores estudios. Claramente esto no es así. Existen recompensas no monetarias, ya sea por gustos, satisfacciones personales en la realización de ciertas tareas, etc. Pero estos beneficios no pueden ser medidos, y no se pueden incorporar a la estimación de los retornos. En este sentido, hay que pensar a los resultados obtenidos como un límite inferior, que sólo incluye la retribución monetaria. Pero existe un componente no monetario no incorporado y en consecuencia el retorno estimado será menor que el real para el individuo.

\section{Conclusiones}

De acuerdo a lo analizado en el trabajo, las tasas de retorno dinámicas son más apropiadas que las estáticas para estimar los retornos a la educación. Primero, porque requieren menos supuestos para que las estimaciones sean interpretadas como retornos a la educación, netos de otros efectos. En este sentido los resultados obtenidos por el método dinámico son más generales. Segundo, porque no infieren los ingresos de un grupo a partir de los ingresos de otro grupo. Esta segunda ventaja toma una gran relevancia cuando los ingresos de los grupos varían a lo largo del tiempo, ya que como se demostró, en estas circunstancias las tasas de retorno estáticas tienden a subestimar (si los ingresos aumnetan) o sobrestimar (si los ingresos disminuyen) los retornos "verdaderos" que surgen de usar los ingresos del grupo analizado a lo largo de su vida laboral.

Para el caso del Gran Buenos Aires, desde 1974 hasta 1999, vimos que debido a la disminución de los ingresos reales que ocurrió en el país durante el período estudiado, las tasas estáticas sobrestiman a las dinámicas en un 2,6\% para el nivel secundario incompleto, $4,6 \%$ para el nivel secundario completo y $4,8 \%$ para el nivel universitario completo. En este sentido, es probable que una persona que tome la decisión de invertir en capital humano y base su decisión en los retornos estáticos esté mirando la señal equivocada, y no obtenga la recompensa esperada al momento de realizar el gasto de la inversión. De acuerdo a los resultados obtenidos, este problema es mayor cuanto más alto el nivel educativo. 
Estos retornos son una cota inferior, debido a que durante todo el análisis se supuso que los únicos beneficios de educarse consistían en ingresos monetarios más elevados. Sin embargo, las personas tienen en cuenta a la hora de tomar sus decisiones variables no monetarias, como la stisfacción personal, o el gusto por realizar una tarea determinada. Pero estos beneficios no pueden ser medidos, y por lo tanto los beneficios incluídos en este trabajo son menores a los efectivamente recibidos. En consecuencia, los retornos estimados son inferiores a los percibidos por los agentes.

Los resultados presentados en este trabajo pueden ser extendidos. Con información más detallada, se podrían incorporar los costos directos de estudiar y se podría determinar con mayor exactitud la duración de los estudios (para cada nivel) para un estudiante promedio, y de esa forma calcular con más precisión los costos de oportunidad. Por último, cuando las encuestas de hogares abarquen un período suficientemente largo, se podrán obtener el perfil de ingresos completo para una cohorte dada, sin la necesidad de completar los años iniciales o finales (según la cohorte sea vieja o joven) a partir de datos de otras cohortes. 


\section{Bibliografía}

Adison, S. and Siebert, W. S., The Market for Labor. An Analitical Treatment

Arias, O. And Mc Mahon, W., Dynamics Rates of Returns to Education in The U.S.. Economic of Education Review, 1999.

Becker, G., Human Capital. NBER, 1964.

Ben Porath, Y., The Production of Human Capital and The Life Cycle of Earnings. Journal of Political Economy, 1967, 75, pp. 352-365.

Card, D., Earnings, Schooling and Ability Revisited. NBER Working Paper 4832, 1994.

Card, D., Estimating the Returns to Schooling: Progress on Some Persistent Econometric Problems. NBER Working Paper 7769, June 2000.

Card, D. and Lemieux, T., Can Falling Supply Explain the Raising Rturn to College for Younger Men? A Cohort Based Analysis. NBER Working Paper 7655, April 2000.

Chiswick, B., Interpreting the Coefficient of Schooling in the Human Capital Earning Function. 1997.

Deaton, A., The Analysis of Household Surveys, 1998

Encuesta Permanente de Hogares, INDEC. Varias ondas, Región Metropolitana

Friedman, M., Teoría de los Precios. Editorial Alianza, 1966.

García Swartz, D., Argentine Wage Differentials, 1974 - 1996. PhD. Thesis, University of Chicago, 1996.

Japelli, T., The Age-Wage Profile and the Life Cycle Hypothesis: A Cohort Analysis With a Time Series Cross-Section of Italian Households. Working paper $N^{o} 14$, Centre Studi in Economia e Finanza, Univesita Degli Studi di Salerno, January 1999.

Hartoog, J., Pereira, P. and Vieira, J., Changing Returns to Education in Portugal During the 1980s and Early 1990s: OLS and Quantile Regression Estimators.

Llach, J. J., Montoya, S. y, Educación para todos. IERAL, 2000.

Lachler, U., Education and Earnings Inequality in Mexico. World Bank 1997.

Layard, R. and Walters, W. ,Microeconomic Theory. Ed. Mc Graw Hill, 1978

Maani, S., Private and Public Returns to Investment in Secondary and Higher Education in New Zealand Over Time: 1981 - 1996. Treasury Working Paper 99/2, New Zealand, 1998.

Mincer, J., Scooling, Experience and Earnings. New York, NBER, 1974

Mock, P., Patrinos, H. and Venkataraman, M., Education and Earnings in a Transition Economy: The Case of Vietnam. World Bank, January 1998.

Neal, D. and Rosen, S., Theories of the Distribution of Labor Earnings. NBER Working Paper 6378, 1998.

Patrinos, H., Market Forces in Education. Word Bank, July 1999.

Psacharopoulos, G., Returns to Investment in Education: A Global Update. World Development, 22, pp. 1325 - 1343. 1994

Psacharopoulos, G.,The Profitability of Investment in Education: Concepts and Methods. World Bank, December 1995

Pesino, C. ,(1995): Inflation and Returns to Education in Grater Buenos Aires 1986 1993: From Hyperinflaion to Stabilization. CEMA, N 104, June 1995.

Rosen, S., Human Capital, A Survey of Empirical Research. Research in Labor Economics, Vol. 1, pp. 3 - 39. Ed. R. Ehrenberg, 1977. 
Willis, R. J., Wage Determinants: A Survey and Reinterpretation of Human Capital Wage Functions. In O. Ashenfelter and R. Layard (eds.). Handbook of Labor Economics, 1986, pp. 525 - 602. Amsterdam, North Holland. 